\title{
Asymptotic Error-Correcting Performance of Joint Source-Channel Schemes based on Arithmetic Coding
}

\author{
Salma Ben-Jamaa \\ LSS - CNRS - Supélec \\ Université Paris-Sud, 11 \\ 3 rue Joliot-Curie - 91192 \\ Gif-sur-Yvette, France \\ Email: salma.ben-jamaa@1ss.supelec.fr
}

\author{
Claudio Weidmann \\ ftw. Telecommunications Research \\ Center Vienna \\ Donau-City-Strasse 1, A-1220 \\ Vienna, Austria \\ Email: weidmann@ftw.at
}

\author{
Michel Kieffer \\ LSS - CNRS - Supélec \\ Université Paris-Sud, 11 \\ 3 rue Joliot-Curie - 91192 \\ Gif-sur-Yvette, France \\ Email: michel.kieffer@1ss.supelec.fr
}

\begin{abstract}
In joint source-channel (JSC) schemes based on arithmetic coding (AC), additional redundancy may be introduced in order to reduce transmission errors. The purpose of this work is to provide analytical tools to predict and evaluate the effectiveness of that redundancy. Integer binary $\mathrm{AC}$ is modeled by a reduced-state automaton in order to obtain a bit-clock trellis of the $\mathrm{AC}$. Considering $\mathrm{AC}$ as a trellis code, distance spectra are then derived. In particular, an algorithm to compute the free distance of an arithmetic code is proposed. The obtained code properties allow to compute upper bounds on both bit error and symbol error probabilities and thus provide an objective criterion to analyze the behavior of JSCAC schemes when used on noisy channels.
\end{abstract}

\section{INTRODUCTION}

Arithmetic Coding [1] is currently being deployed in a growing number of applications as it yields higher compression efficiency when compared to other compression methods. However, the high compression rate makes AC particularly vulnerable to transmission errors. This issue has motivated the recent development of Joint Source-Channel (JSC) techniques for AC-encoded data [2]-[7].

Improving the robustness of AC against transmission errors is usually achieved by introducing redundancy in the compressed bitstream. In [2], Boyd et al. introduced a forbidden symbol (FS) in the source alphabet and used it at the decoder side as an error detection device. The effectiveness of this technique was analyzed by Chou et al. in [4], where the FS was used to achieve continuous error detection, and ARQ was implemented for error correction. Sayir [3] considered the arithmetic coder as a channel coder and added redundancy in the transmitted bitstream by introducing gaps in the coding space; he proposed to use the stack sequential decoding algorithm. In [5], Pettijohn et al. used both depth-first and breadth-first sequential decoding, where error detection is achieved by testing the presence of a FS in the decoded bitstream. Grangetto et al. [7] proposed a MAP decoder of AC using the FS. In [8], Demiroglu et al. used Trellis Coded Modulation (TCM) jointly with AC; the FS was exploited in order to discard erroneous paths during a List Viterbi decoding process. In [6], Guionnet et al. viewed a quasi-arithmetic coder as a state machine where transitions between states were modeled by a Markov process. A symbol-clock and a bit-clock trellis were proposed, and redundancy was added by limiting the number of states and introducing synchronization markers.

In this paper, we consider a practical integer-based implementation of AC for a memoryless source, and we derive a trellis representation of the coding process. Based on this representation, the errorcorrecting performance can be characterized using distance properties of the code. This is explained in [9] for convolutional codes, and in [10] for Variable Length Error Correcting (VLEC) codes. To the best of our knowledge, no similar approach has been carried out for arithmetic codes. In this paper, tests have been performed using AC where some probability is alloted to a FS. However, we emphasize that the proposed approach is applicable to all methods of adding redundancy to AC implemented with a finite-state machine.

Section II introduces the basic principles of arithmetic coding and the integer implementation of AC. Section III, explains how AC can be viewed as a finite state machine, and presents a reduced-state trellis used for encoding and decoding. In Section IV, distance properties and error bounds are derived and a practical algorithm allowing to compute the free distance of $\mathrm{AC}$ is proposed. Finally, simulation results are shown in Section V.

\section{INTEGER ARITHMETIC CODING}

$\mathrm{AC}$ is based on recursive subdivision of the interval $[0,1)$ into non-overlapping source intervals whose widths are equal to the probabilities of the corresponding source sequences. In the case of binary $\mathrm{AC}$, the current source interval [low, high) is partitioned into two subintervals, the widths of which are proportional to the probabilities $P_{0}$ and $P_{1}$ of the source symbols 0 and 1 , respectively. One of these intervals is selected as the new source interval, according to the value of the current symbol. Once the last symbol is encoded, the encoder computes the code interval $\left[v 2^{-l},(v+1) 2^{-l}\right) \subset[$ low, high $)$, such that $v$ is an integer from $\left\{0,1, \ldots, 2^{l}-1\right\}$, and $l$ is the minimum number of bits needed to represent the size of the last interval $[$ low, high). For sources with skewed probabilities, and for long source sequences, subintervals may get too small to be accurately handled by a finite precision computer.

This problem is solved by integer binary $\mathrm{AC}$, where the interval $[0,1)$ of reals is replaced by the interval $[0, T)$ of integers, $T=2^{p}$, $p \geq 2$ being the size in bits (precision) of the initial interval. Partition and selection are carried out every time a source symbol is encoded. Renormalization by doubling the source interval size is performed if one of the following conditions holds

1) If low $<2^{p-1}$, low and high are doubled.

2) If $2^{p-1} \leq$ high, low and high are doubled after subtracting $2^{p-1}$.

3) If $2^{p-2} \leq$ low and high $<3 \times 2^{p-2}$, low and high are doubled after subtracting $2^{p-2}$.

If the current interval (before renormalization) overlaps the midpoint of $\left[0,2^{p}\right)$, no bit is output. The number of consecutive times this occurs is stored in a variable called follow. If the current interval (before renormalization) lies entirely in the upper or lower half of $\left[0,2^{p}\right)$, the encoder emits the leading bit of low (0 or 1$)$ and follow opposite bits ( 1 or 0$)$. This is called the follow-on procedure [1].

At the decoder side, a buffer of $p$ bits is formed by the last $p$ received bits. The integer represented by that buffer is used to 
determine the subdivisions and selections within $[0, T)$ which have been performed at the encoder side.

In the remainder of this paper, symbols will stand for binary inputs of the encoder and bits for its outputs.

\section{TRELlis-BASED ARITHMETIC CODING AND DECODING}

The integer binary AC can be considered as an automaton and represented by a finite number of states and transitions, except for the possibly unbounded follow counter. This idea was first proposed by Howard et al. in [11], where arithmetic operations were replaced by table lookups. Howard et al. observed that by rounding the interval boundaries, one can noticeably decrease the computational cost of AC without significantly degrading the compression performance. In [6], the table-lookup representation was exploited in order to derive a stochastic automaton which is then followed by a convolutional code. Iterative decoding was used at the receiver side. Recently, integer arithmetic decoding was represented by a 3D trellis taking into account the presence of a FS in the source alphabet [12].

\section{A. AC interpreted as a state machine}

When performing integer $\mathrm{AC}$, the number of possible subintervals of $[0, T)$ is limited by $3 T^{2} / 16$. On the other hand, for a memoryless source with a known probability model, the encoder is entirely characterized by the current interval [low, high) and the value of follow. Hence, the encoder state can be represented by (low, high, follow) as defined in [6]. The idea is thus to precompute all possible states of the arithmetic encoder such that any source stream may be encoded using table lookups rather than arithmetic operations. However, as the variable follow may grow without bound in principle, the number of states (low, high, follow) could be infinite.

In order to cope with the uncontrolled growth of follow, we choose to keep it below a given threshold $F_{\max }$. Indeed, when follow $=F_{\max }$, the symbol probabilities are modified such that a follow-on procedure is performed as soon as the next symbol is encoded. Let $\left[\operatorname{low}_{f}, h i g h_{f}\right)$ be the current interval, such that $\frac{T}{4} \leq l o w_{f}<\frac{T}{2}$ and $\frac{T}{2}<h_{i g h}<\frac{3 T}{4}$. This interval is subdivided into $\left[\operatorname{low}_{f}, \frac{T}{2}\right)$ and $\left[\frac{T}{2}, h i g h_{f}\right)$. The first subinterval is selected if the next symbol is 0 , the second is selected if the next symbol is 1 .

The states of the arithmetic coder are determined recursively. Starting at the initial state $(0, T, 0)$, the encoder is fed by all possible inputs, and may either reach an already known state or a new one. This exhaustive search stops when no new state is found. Figure 1 shows the finite state machine (FSM) representing an integer AC with $T=16, P_{0}=2 / 11$ and $F_{\max }=1$. Input symbols and output bits are marked on each transition. When no bits are output by the encoder, the transition label is written as $1 /-$ or $0 /-$.

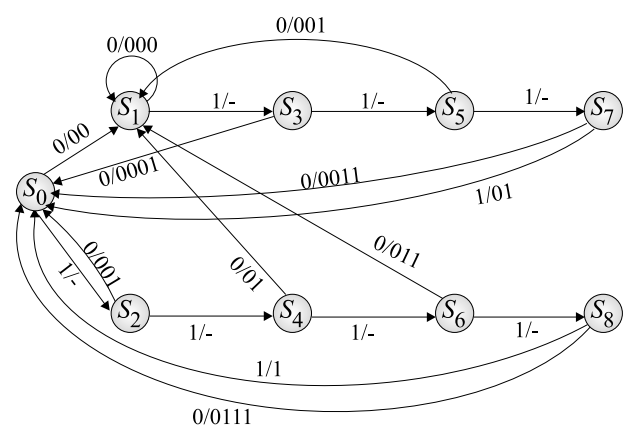

Fig. 1. FSM obtained for $T=16, P_{0}=2 / 11, F_{\max }=1$.

\section{B. Reduced state machine}

As it can be seen on the FSM in Figure 1, several transitions are such that no bits are output by the encoder. These are called mute transitions. As our first goal is to represent the arithmetic coder by a bit-clock trellis, one solution is to consider the 3D trellis including transitions between states positioned at the same depth as in [12]. Here, transitions are allowed to have more than a single input symbol, indeed, every mute transition is extended until at least one bit is output. The algorithm of transition extension is explained below.

Consider $\mathcal{S}_{\mathrm{FSM}}$, the set of all states of a FSM, and $\mathcal{T}_{\mathrm{FSM}}$, the set of all transitions between states. Let $\mathcal{T}_{x y}$ be the set of transitions between state $S_{x}$ to state $S_{y}$. Let $t_{x y}$ be an element of $\mathcal{T}_{x y}$. The input symbols and the output bits associated to $t_{x y}$ are respectively denoted by in $\left(t_{x y}\right)$ and out $\left(t_{x y}\right)$. If $t_{x y}$ is extended with a transition $t_{y z}$, a new transition $t_{x z}$ is created to replace $t_{x y}$. Then, $i n\left(t_{x z}\right)$ is the concatenation of $i n\left(t_{x y}\right)$ and $i n\left(t_{y z}\right)$, and out $\left(t_{x z}\right)$ is the concatenation of out $\left(t_{x y}\right)$ and out $\left(t_{y z}\right)$.

Let $\mathcal{T}_{\text {mute }}$ be the subset of $\mathcal{T}_{\text {FSM }}$ containing all the mute transitions. Algorithm 1 in Table I described how the mute transitions may be removed from a FSM to obtain an equivalent FSM without mute transitions.

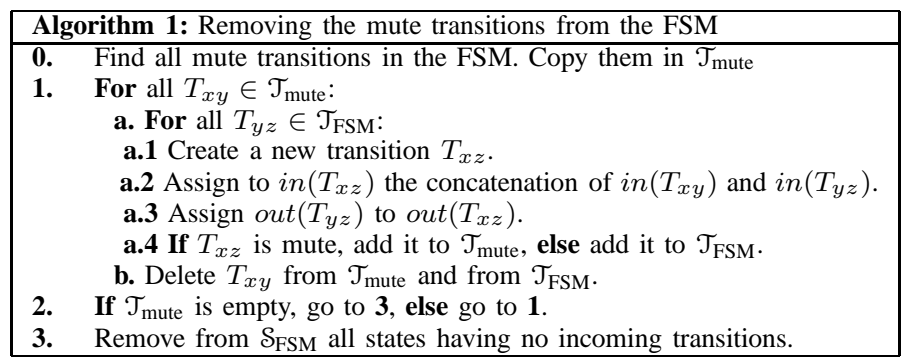

TABLE I

CONSTRUCTION OF THE REDUCED FSM

In some cases, $\mathcal{S}_{\mathrm{FSM}}$ may contain states with a single incoming transition or a single outgoing transition. The algorithm of Table I may also be applied in order to remove such states from the graph of the FSM. The reduced FSM obtained from the example of Figure 1 is depicted in Figure 2.

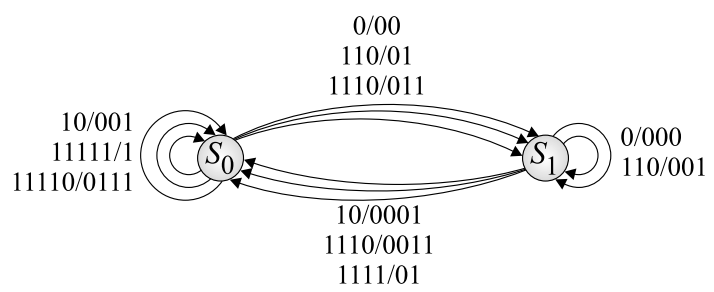

Fig. 2. Reduced FSM graph obtained for $T=16, P_{0}=2 / 11, F_{\max }=1$.

The resulting reduced FSM is used to generate a bit-clock trellis, with transitions having variable length inputs and outputs. The trellis generated from the reduced FSM of Figure 2 is shown in Figure 3.

\section{Coding and decoding process}

Here, encoding and decoding are entirely based on the trellis representation of AC. The encoder always starts at $S_{0}=(0, T, 0)$. From any given state, the input sequences determining the transitions to the next states are prefix-free, thus the encoding process is instantaneous. When encoding the last symbols of the source sequence, the 


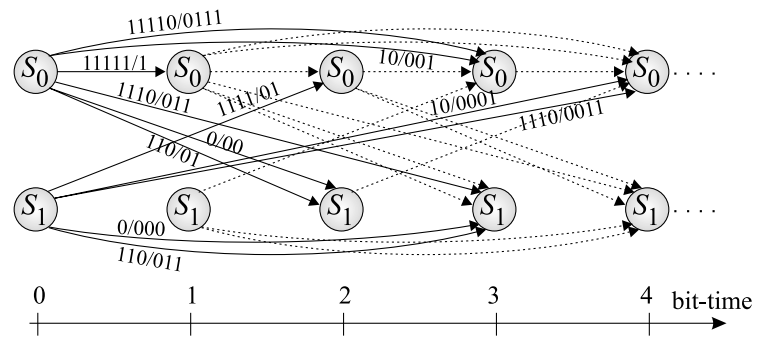

Fig. 3. Example of bit-clock trellis representation of integer binary AC $\left(T=16, P_{0}=2 / 11, F_{\max }=1\right)$

encoder may have the choice between several transitions and thus possibly several final states. In that case, the encoder chooses the transition having the shortest output. This termination may insert some additional bits in the output bitstream, but in practice, the number of additional bits is very close to the number of bits added by an encoder based on a lookup table. On the other hand, decoding on the proposed reduced trellis is not instantaneous as the output bit sequences of transitions are not prefix-free in general. A List Viterbi algorithm on the obtained reduced trellis is used for decoding. Unlike the trellis proposed in [12], which needs a third dimension to keep track of mute transitions, here the constructed two dimensional trellis is sufficient to run the Viterbi decoder.

\section{Redundancy introduced by a FS}

As mentioned in Section I, a well known JSC technique with AC is based on the introduction of a particular symbol in the source alphabet. This symbol is called the forbidden symbol (FS) because it is never sent by the source, although a positive probability $P_{\varepsilon}$ is allocated to it. In that way, the decoding of the FS indicates the occurrence of a transmission error. It can be shown that introducing a forbidden symbol of probability $P_{\varepsilon}$ adds a redundancy of $-\log (1-$ $\left.P_{\varepsilon}\right)$ bits/symbol to the coded bitstream. The trellis associated to an AC may be derived in the same way whether a FS is used or not. The coding and decoding processes are performed on the reduced trellis as explained in Section III-C. The effects of the redundancy introduced by the FS appear naturally in the trellis representation and can be characterized by the distance properties explained in the next section.

\section{Performance analysis of trellis-Based AC}

As established in Section III, the FSM interpretation leads naturally to consider the integer arithmetic code as a trellis code. The performance of trellis codes on channels with moderate to large signal-tonoise ratios (SNR) can be predicted from their distance properties [13].

Let $\mathbf{p}_{x y}^{n}$ be a path of $n$ bits on the trellis, starting from $S_{x}$ and ending in $S_{y}$. As we consider a bit-clock trellis (see Figure 3), if $S_{x}$ is at bit-time 0 , then $S_{y}$ is at bit-time $n$. The Hamming distance between two paths $\mathbf{p}_{x y}^{n}$ and $\mathbf{q}_{x y^{\prime}}^{n}$ of the same length $n$ and with the same initial state $S_{x}$ is denoted by $d_{H}\left(\mathbf{p}_{x y}^{n}, \mathbf{q}_{x y^{\prime}}^{n}\right)$. When a path $\mathbf{p}_{x y}^{n}$ is extended by a transition $t_{y z}$, it becomes $\mathbf{p}_{x z}^{n^{\prime}}$. The new length $n^{\prime}$ is such that $n^{\prime}=n+\ell\left(\right.$ out $\left.\left(t_{y z}\right)\right)$, $\ell$ being the length function.

\section{A. Free distance}

At the decoder side, when a trellis-based Viterbi algorithm is used, any two distinct paths that start at a given state and end in another represent received sequences that may be confused by the decoder. The closest paths determine the worst case for error detection. The distance between the closest paths is called the free distance $d_{\text {free }}$.
For linear codes, such as convolutional codes, the free distance is equal to the minimum Hamming weight of paths diverging from then remerging to the all zero path [9]. In the case of non-linear codes (such as VLEC and arithmetic codes), comparing paths to the all zero path may not be sufficient to determine the free distance since the codes are generally not geometrically uniform [14]. In [10], Buttigieg deduced a lower bound of the free distance from the distance between all possible pairs of unequal length VLEC codewords. Extending this technique to $\mathrm{AC}$ assumes that the outputs of the transitions are VLC codewords. However, these outputs may not satisfy the prefix condition in general (see Figure 3), making the lower bound defined in [10] equal to zero. Here, we propose an algorithm for computing the free distance for a trellis-based AC. This algorithm relies on an iterative computation of the smallest distances between all different paths of the same length starting from the same state.

For computational purposes, it is advantageous to introduce intermediate states such that every state transition outputs exactly one bit. If a given transition outputs two bits, it is divided into two transitions, the first inherits the input symbols and the first output bit, the second has no input symbol and outputs the second bit. Notice that introducing those states does not increase the total number of possible paths through the trellis given that every intermediate state has a single incoming transition and a single outgoing transition. Let $\mathcal{S}_{\mathrm{FSM}}^{\prime}$ be the set of $N_{S}^{\prime}$ states in the newly constructed FSM, containing all states of $\mathcal{S}_{\mathrm{FSM}}$ and the intermediate states.

The free distance evaluation uses a three dimensional array $D_{n}$ defined as follows: for $y \neq z, D_{n}(x, y, z)$ is the minimum distance between all pairs of paths of length $n$, starting at $S_{x}$, ending respectively in $S_{y}$ and $S_{z}$, and never converging. For $z=y$, $D_{n}(x, y, y)$ corresponds to the minimum distance between pairs of paths of at most $n$ bits, diverging at state $S_{x}$ and converging for the first time at state $S_{y}$. Let $\mathcal{G}_{n}(x, y)$ be the set of all pairs of paths diverging from the same starting state $S_{x}$ and converging for the first time $n$ bits later to $S_{y}$. Let $\mathcal{G}_{n}(x)$ be the set of all pairs of paths of length $n$ bits, diverging from the starting state $S_{x}$ and never converging. One gets:

$$
\begin{aligned}
& D_{n}(x, y, z)=\min _{\left(\mathbf{p}_{x y}^{n}, \mathbf{q}_{x z}^{n}\right) \in \mathcal{S}_{n}(x)} d_{H}\left(\mathbf{p}_{x y}^{n}, \mathbf{q}_{x z}^{n}\right), \text { if } y \neq z(1) \\
& D_{n}(x, y, y)=\min _{n^{\prime} \leq n} \min _{\left(\mathbf{p}_{x y}^{n^{\prime}}, \mathbf{q}_{x y}^{n^{\prime}}\right) \in \mathcal{G}_{n^{\prime}}(x, y)} d_{H}\left(\mathbf{p}_{x y}^{n^{\prime}}, \mathbf{q}_{x y}^{n^{\prime}}\right)
\end{aligned}
$$

The free distance is thus computed as $d_{\text {free }}=\min _{n, x, y} D_{n}(x, y, y)$.

For initializing $D_{n}$ at bit-time 1 , all the transitions starting at $S_{x} \in$ $\mathcal{S}_{\mathrm{FSM}}$ and ending in $S_{y} \in \mathcal{S}_{\mathrm{FSM}}^{\prime}$ are used to compute values of $D_{1}$ such that

$$
D_{1}(x, y, z)=\min _{\substack{T_{x y} \in \mathcal{T}_{x y}, T_{x z} \in \mathcal{T}_{x z} \\ t_{x z} \neq \mathcal{T}_{x z}}} d_{H}\left(t_{x y}, t_{x z}\right) .
$$

By convention, if one of the sets $\mathcal{T}_{x y}$ or $\mathcal{T}_{x z}$ is empty, $d_{H}\left(t_{x y}, t_{x z}\right)=$ $+\infty$. Notice that $x$ cannot index an intermediate state since these states have a single outgoing transition, consequently the trellis cannot contain two paths diverging at an intermediate state.

At each iteration, $D_{n}$ is deduced from $D_{n-1}$ as follows

$$
\begin{aligned}
& D_{n}(x, y, z)=\min _{y^{\prime}, z^{\prime} \in Q_{y, z}}\left\{D_{n-1}\left(x, y^{\prime}, z^{\prime}\right)+d_{H}\left(t_{y^{\prime} y}, t_{z^{\prime} z}\right)\right\} \\
& \text { if } y \neq z, \\
& \begin{aligned}
D_{n}(x, y, y)= & \min _{y^{\prime}, z^{\prime} \in Q_{y, z}}\left\{D_{n-1}(x, y, y), D_{n-1}\left(x, y^{\prime}, z^{\prime}\right)\right. \\
& \left.+d_{H}\left(t_{y^{\prime} y}, t_{z^{\prime} y}\right)\right\} \quad \text { else, }
\end{aligned}
\end{aligned}
$$


where $Q_{y, z}=\left\{y^{\prime} \neq z^{\prime} \in\left\{1, \ldots, N_{S}^{\prime}\right\}: t_{y^{\prime} y} \in \mathcal{T}_{y^{\prime} y}, t_{z^{\prime} y} \in \mathcal{T}_{z^{\prime} y}\right\}$.

The algorithm computing the free distance on the trellis is summarized in Table II.

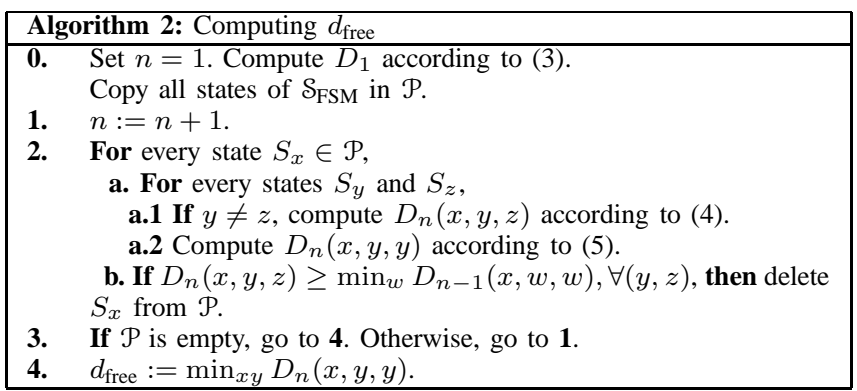

TABLE II

FREE DISTANCE COMPUTATION

The stop condition of Algorithm 2, at steps 2.b and 3. is based on the following observation. Consider $\mathbf{p}_{x y}^{n}$ and $\mathbf{p}_{x y^{\prime}}^{n}$, two paths of length $n$ starting at the same state $S_{x}$. Let $d_{n}$ be the Hamming distance between these paths. If the extensions of these paths converge for the first time at bit-time $n^{\prime}>n$, then the Hamming distance $d_{n^{\prime}}$ between the converging paths is such that $d_{n^{\prime}} \geq d_{n}$. Therefore, if a pair of paths has a Hamming distance larger than the one already found between shorter converging paths starting at the same state, there is no need to consider the extensions of that pair of paths. Hence, when all pairs of paths of length $n$ are at a Hamming distance greater than the minimum found between two shorter converging paths, the algorithm stops and $d_{\text {free }}$ is computed (see step 4., Table II).

\section{B. Distance spectrum}

In order to evaluate the distance spectrum, the analysis made in [10] on VLECs can be translated to the AC. Two quantities are required: $A_{h}$, the average number of converging pairs of paths at Hamming distance $h$, and $B_{h}$, the average Levenshtein distance $d_{L}$ [15] between variable-length input sequences of all converging pairs of paths whose output bistreams are at Hamming distance $h$ :

$$
\begin{aligned}
A_{h} & =\sum_{n=1}^{\infty} \sum_{\substack{\left(\mathbf{p}_{n}, \mathbf{q}_{n}\right) \in \mathcal{G}_{n}: \\
d_{H}\left(\mathbf{p}_{n}, \mathbf{q}_{n}\right)=h}} P\left(\mathbf{p}_{n}\right) . \\
B_{h} & =\sum_{n=1}^{\infty} \sum_{\substack{\left(\mathbf{p}_{n}, \mathbf{q}_{n}\right) \in \mathcal{G}_{n}: \\
d_{H}\left(\mathbf{p}_{n}, \mathbf{q}_{n}\right)=h}} P\left(\mathbf{p}_{n}\right) d_{L}\left(i n\left(\mathbf{p}_{n}\right), i n\left(\mathbf{q}_{n}\right)\right) .
\end{aligned}
$$

where $P\left(\mathbf{p}_{n}\right)$ denotes the a priori probability of the path $\mathbf{p}_{n}$, which is supposed to be the correct path, and $\mathcal{G}_{n}$ is the set of all pairs of paths diverging from the same starting state and converging for the first time $n$ bits later.

For the evaluation of $A_{h}$ and $B_{h}$ in [10], only paths beginning at a single initial state (corresponding to bit-time $n=0$ ) had to be considered. In our case all the states of the reduced trellis have to be treated as starting states. Hence, the a priori probability of a given path $P\left(\mathbf{p}_{x y}^{n}\right)$ is evaluated by means of the a priori source probabilities, and the probability $P\left(S_{x}\right)$ of being at the state $S_{x}$. One gets

$$
\left.P\left(\mathbf{p}_{x y}^{n}\right)=P\left(S_{x}\right) P\left(i n\left(\mathbf{p}_{x y}^{n}\right)\right)\right)
$$

Assuming that paths are very long and that the source is stationary, the probability $P\left(S_{x}\right)$ of being at a given state can be evaluated by means of the Markov transition matrix $M_{T}$ corresponding to the reduced FSM [16]. $M_{T}(x, y)$ is the probability that the next state is $S_{y}$, knowing that the current one is $S_{x}$ :

$$
M_{T}(x, y)=\sum_{t_{x y} \in \mathcal{T}_{x y}} P\left(t_{x y} \mid S_{x}\right)=\sum_{t_{x y} \in \mathcal{T}_{x y}} P\left(i n\left(t_{x y}\right)\right) .
$$

The sought distribution $P\left(S_{x}\right)$ is the stationary distribution of the Markov chain defined by $M_{T}$ (so far we have encountered no examples where this Markov chain is not ergodic). Pairs of paths belonging to $\mathcal{G}_{n}$ may be identified using one of the two algorithms proposed in [10] applied on every possible starting state separately.

\section{Error bounds}

The same error bounds as for convolutional codes [9] were found by Buttigieg [10] in the case of non-linear VLEC trellises. Applying similar reasoning on arithmetic coding trellises leads to the same error bound expressions, although the derivation of distance properties is different. Hence, let $P_{e r}^{b}$ be the error event probability at any bit position, and $P_{e r}^{s}$ be the error event probability at any source symbol position. These error probabilities are bounded by

$$
\begin{aligned}
& P_{e r}^{b} \leq \sum_{h=d_{\text {free }}}^{\infty} A_{h} P_{h} \\
& P_{e r}^{s} \leq \sum_{h=d_{\text {free }}}^{\infty} B_{h} P_{h},
\end{aligned}
$$

where $P_{h}$ is the probability that an erroneous path at Hamming distance $h$ is selected by the decoder instead of the correct path. Assume that encoded bits are mapped onto symmetric BPSK signals of amplitude $\pm \sqrt{E_{b}}$. For an AWGN channel, the probability $P_{h}$ can be written as :

$$
P_{h}=\frac{1}{2} \operatorname{erfc} \frac{\sqrt{h \times E_{b}}}{\sigma \sqrt{2}},
$$

were $\sigma$ is the variance of the zero-mean Gaussian noise [17].

\section{EXPERIMENTAL RESULTS AND DISCUSSION}

The encoder used for testing the error-correcting performance is characterized by four parameters: $p$ defining the bit size of the initial interval $[0, T), P_{0}$ defining the probability used by the encoder to code the symbol $0, F_{\max }$ being the chosen upper limit of the variable follow, and $P_{\varepsilon}$ the probability of the FS. The probability space allocated to the FS is [low, low + range $\left.\cdot \frac{P_{\varepsilon}}{2}\right) \cup[$ high - range . $\frac{P_{\varepsilon}}{2}$, high).

We use a random binary source with $P(0)=\frac{1}{8}$. Table III shows the additional redundancy introduced due to the limitation of follow, computed as $\frac{\text { average length-entropy }}{\text { entropy }} \times 100$, where average length is the number of bits/symbol is the code bitstream. One can notice that bounding follow has a limited effect on the compression rate. Indeed, in the worst case $\left(F_{\max }=1\right)$, the difference between the code average length and the entropy is $0.0282 \mathrm{bit} / \mathrm{symbol}$. The same table compares the number of states obtained when performing the exhaustive search of states described in III-A to the number of states in the reduced trellis described in III-B. The number of states can be reduced by a factor of 10 for $F_{\max }=9$, without noticeable loss of compression performance. The reduction of state number allows to reduce the memory required by the Viterbi decoder although the number of transitions, denoted by $N_{T}$, may still be significant.

Figure 4 compares the asymptotic symbol error bound to the symbol error rate (SER) obtained with simulations for different values 


\begin{tabular}{|l|l|l|l|l|}
\hline$F_{\max }$ & 1 & 3 & 6 & 9 \\
\hline \hline Additional redundancy & $5.19 \%$ & $0.95 \%$ & $0.60 \%$ & $0.01 \%$ \\
\hline$N_{S}$ in the exhaustive trellis & 119 & 216 & 302 & 363 \\
\hline$N_{S}$ in the reduced trellis & 26 & 37 & 36 & 36 \\
\hline$N_{T}$ in the reduced trellis & 203 & 440 & 828 & 1404 \\
\hline
\end{tabular}

TABLE III

EFFECT OF THE CHOICE OF $F_{\max }$ ON THE TRELLIS FEATURES,

$$
p=6, P_{\varepsilon}=0
$$

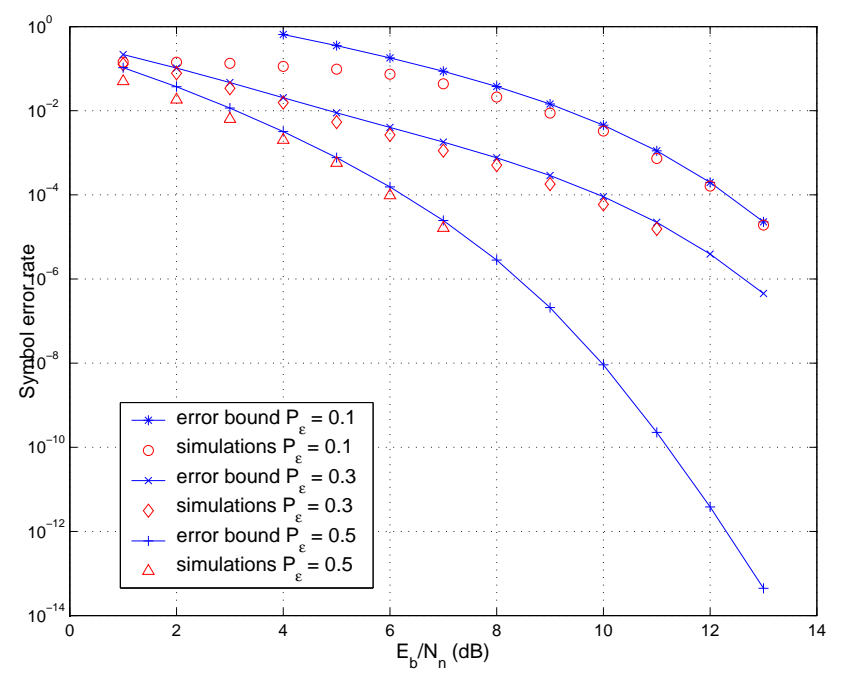

Fig. 4. Simulations and error bounds on the SER for different amounts of additional redundancy

of $P_{\varepsilon}$. Simulations have been performed using source sequences of 512 symbols, and a List Viterbi decoder fed with the noisy modulated bits. Values of $A_{h}$ and $B_{h}$ are computed till $h=10$ and using paths of length up to 22 bits. There is no need to consider longer paths as the probability of a path decreases exponentially with its length. The SER is estimated by means of the Levenshtein distance computed between the initial source sequence and the decoded one. The algorithm computing the Levenshtein distance has been implemented according to [18]. As expected, the bound on the SER is tight at medium to high SNR. In particular, the asymptotic curves allow to predict and compare the behavior of the decoder at high SNR without need of simulations. The trellis features of encoders used to get Figure 4 are summarized in Table IV. Notice that when the amount of introduced redundancy increases, the free distance may increase as in the case of $P_{\varepsilon}=0.5$. When comparing codes obtained with $P_{\varepsilon}=0.1$ and $P_{\varepsilon}=0.3$, we observe that the free distance is equal to 1 in both cases, however, the effect of the redundancy appears in the variation of $B_{d_{\text {free }}}$. Indeed, $B_{d_{\text {free }}}$ decreases when $P_{\varepsilon}$ increases, leading to lower SER.

\section{CONCLUSIONS AND FUTURE WORK}

In this paper, we have proposed analytical tools allowing to characterize the efficiency of the redundancy introduced in arithmetic coding schemes. Indeed, the majority of the techniques of JSC coding related to AC proposed in the literature can be evaluated using the proposed tools, since the introduced redundancy has a direct effect on the distance properties of the derived trellis. Current work is dedicated to finding out the optimal way of introducing redundancy in AC.

\begin{tabular}{|l|l|l|l|}
\hline$P_{\varepsilon}$ & 0.1 & 0.3 & 0.5 \\
\hline \hline$d_{\text {free }}$ & 1 & 1 & 2 \\
\hline$A_{d_{\text {free }}}$ & 1.19 & 0.085 & $9.26 \quad 10^{-5}$ \\
\hline$B_{d_{\text {free }}}$ & 5.73 & 0.114 & $3.1810^{-4}$ \\
\hline$N_{S}$ in the reduced trellis & 7 & 11 & 5 \\
\hline$N_{T}$ in the reduced trellis & 77 & 51 & 25 \\
\hline
\end{tabular}

TABLE IV

EFFECT OF $P_{\varepsilon}$ ON THE TRELlis fEATURES, $p=5, F_{\max }=1$

\section{ACKNOWLEDGMENT}

This work has been partly supported by the NEWCOM NoE. The authors would like to thank Pierre Duhamel for valuable suggestions and comments.

\section{REFERENCES}

[1] I. H. Witten, R. M. Neal, and J. G. Cleary, "Arithmetic coding for data compression," Communications of the ACM, vol. 30(6), pp. 520-540, 1987.

[2] C. Boyd, J. Cleary, I. Irvine, I. Rinsma-Melchert, and I. Witten, "Integrating error detection into arithmetic coding," IEEE Trans. on Comm., vol. 45(1), pp. 1-3, 1997.

[3] J. Sayir, On Coding by Probability Transformation, Ph.D. Thesis Nr. 13099, EE Department, ETH Zurich, Switzerland, 1999.

[4] J. Chou and K. Ramchandran, "Arithmetic coding-based continuous error detection for efficient ARQ-based image transmission," IEEE Trans. on Comm., vol. 18(6), pp. 861-867, 2000.

[5] B. D. Pettijohn, M. W. Hoffman, and K Sayood, "Joint source/channel coding using arithmetic codes," IEEE Trans. on Comm., vol. 49(5), pp. 826-836, 2001.

[6] T. Guionnet and C. Guillemot, "Soft decoding and synchronization of arithmetic codes: Application to image transmission over noisy channels," IEEE Trans. on Image Processing, vol. 12(12), pp. 15991609, 2003.

[7] M. Grangetto, P. Cosman, and G. Olmo, "Joint source/channel coding and MAP decoding of arithmetic codes," IEEE Trans. on Comm., vol. 53(6), pp. 1007-1016, 2005.

[8] C. Demiroglu, W. M. Hoffman, and K. Sayood, "Joint source channel coding using arithmetic codes and trellis coded modulation," Proc. of DCC, Snowbird, Utah, USA., pp. 302-311, 2001.

[9] S. Lin and D.J. Costello, "Error control coding: Fundamentals and applications," Englewood Cliffs, Prentice-Hall.

[10] V. Buttigieg, Variable-Length Error Correcting Codes, Ph.D. Thesis, EE Department, Univ. Manchester, Manchester, U.K., 1995.

[11] P. G. Howard and J. S. Vitter, "Practical implementations of arithmetic coding," Image and Text Compression, vol. 13(7), pp. 85-112, 1992.

[12] B. Dongsheng, W. Hoffman, and K. Sayood, "State machine interpretation of arithmetic codes for joint source and channel coding," Proc. of DCC, Snowbird, Utah, USA., pp. 143-152, 2006.

[13] M. Rouanne and D. J. Costello, "An algorithm for computing the distance spectrum of trellis codes," IEEE Trans. on Information Theory, vol. 7(6), pp. 929-940, 1989.

[14] G. D. Forney, "Geometrically uniform codes," IEEE Trans. on Information Theory, vol. 37(5), pp. 1241-1260, 1991.

[15] V. I. Levenshtein, "Binary codes with correction of deletions, insertions and substitution of symbols," Dokl. Akad. Nank. SSSR, vol. 163, pp. $845-848,1965$.

[16] T. M. Cover and J. A. Thomas, Elements of Information Theory, Wiley Series in Telecommunications, 1991.

[17] A. J. Viterbi and J.K. Omura, "Principles of digital communication and coding," McGraw-Hill, New-York, USA., 1979.

[18] Y. Takishima, M. Wada, and H. Murakami, "Error states and synchronization recovery for variable length codes," IEEE Trans. on Comm., vol. 42, pp. 783-792, 1994. 